\title{
Coverage-enhancing Algorithm Based on Priority in WMSN
}

\author{
Zhe-Yuan Xiong, Luo-Na Nie, Jiang-Shan Zhang, Xiao-Min Xiong \\ Department of Public Security Technology, \\ Jiangxi Police Institute, \\ Nanchang, China \\ E-mail: xiongzhey@126.com
}

\begin{abstract}
Coverage area and network lifetime are major problems in wireless multimedia sensor networks (WMSN). In order to optimize the network coverage and maximize the network's lifetime, we propose a coverage-enhancing algorithm based on sensors' priority. To increase the lifetime of the network, the density of distributed sensors could be such that a subset of sensors can cover the required air space. The sensors are prioritized based on their visual and Communicative properties and the selection is performed according to the prioritizing function. Simulation results show that the proposed algorithm achieves more effective enhancement on network coverage compared to the existing algorithm.
\end{abstract}

Keywords-Coverage enhancement; network lifetime; priority; wireless multimedia sensor networks

\section{INTRODUCTION}

Wireless multimedia sensor networks (WMSN) consist of tiny camera nodes, which integrate the image sensor, embedded processor, and wireless transceiver ${ }^{[1]}$. Images are captured and compressed by the camera nodes, and then sent to the base station or sink. The convenience of deployment and the capability to communicate via wireless links made them attractive in many applications, such as monitoring system, intrusion detection, and people tracking. In general, WMSN differs from traditional wireless sensor network in some fundamental aspects. Energy optimization needs to take into account both computation and communication required in a given application. A critical consideration in designing WMSN is conserving energy to enable untethered and unattended operation for an extended period of time.

When it comes to surveillance of large areas, such as parking lots, public areas, and large stores, the complete coverage of the area at all times is required ${ }^{[2]}$. Therefore, seamless coverage of a vast area is an important issue in these applications. After randomly deployed, coverageenhancing algorithm can maximize coverage of a region with minimum activated sensors and maximize networks lifetime with maximum redundant sensors.

\section{RELATED WORKS}

In the recent past, a number of approaches have been significantly used by researchers to enhance the coverage percentage and prolong the network lifetime in WMSN. Yildiz et al. addressed the problem of sensor nodes deployment in heterogeneous WMSN to provide video panorama with a tradeoff between cost and resolution ${ }^{[3]}$. The problem is modeled as mixed-integer program which solves the problem in two phases, viz., master problem and sub problem in a recursive manner. Li et al. [4] proposed a twotier heterogeneous node deployment strategy to prolong lifetime of a WMSN while minimizing cost and limiting the effects of the energy hole problem. The visual sensor nodes are deployed uniformly in first tier for capturing images, whereas the other nodes are deployed using Gaussian distribution in second tier for relaying received images from visual sensor nodes.

In the case of obstacles existed in sensing field, node's orientation was determined by a distributed algorithm to minimize the effect of occlusions and total overlapping regions. The solution based on a bi-level mixed integer program was used to provide full multi-perspective coverage with fewer nodes, which paid less attention to overlapping coverage. For given region, several algorithms have been presented to optimize network coverage based on virtual force. Tao et al. translated the coverage problem into the centroid points' uniform distribution problem ${ }^{[5]}$. Each node adjusted direction with same angle to reduce the overlapping regions and increase the coverage of sensing blanks. The algorithm was failed to fully consider different influences with different overlapping regions. Paired tangent point repulsion mechanism was utilized to modify the above algorithm with the reduction of nodes' computation, whereas it could not increase the coverage much more owing to merely focusing on the nearest node ${ }^{[6]}$. The two algorithms mentioned above did not consider the prolongation of network lifetime.

The objective of sensor coverage problems is to conserve energy usage by minimizing the number of active sensors. The selected sensors should form sufficient coverage of the intended region. Various parameters have been considered by different solutions in the literature, including energy efficiency and bandwidth allocation issues.

\section{MULTIMEDIA SENSOR NETWORKS SCENARIO}

In a multimedia sensor network, multiple camera sensors are deployed to provide multiple views, multiple resolutions, and enhanced observations of the environment ${ }^{[7]}$. These multiple cameras possibly have overlapped FoV(Field of View), as illustrated in Fig.1. The FoV of a camera can be formally defined as a circular sector of extension dependent on the camera angular width, and oriented along the pointing direction of the camera ${ }^{[8]}$. A camera's FoV is determined by four parameters, where $\mathrm{P}$ is the location of the camera, $\mathrm{R}$ is 
the sensing radius, $\mathrm{V}$ is the sensing direction, and $\alpha$ is the offset angle. A given FoV typically encompasses static or moving objects at different depths positioned in front of a far-field still background. An example scenario of WMSN is shown in Fig. 2.

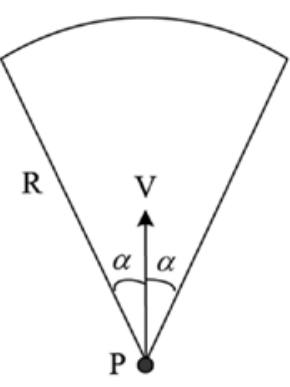

(a)

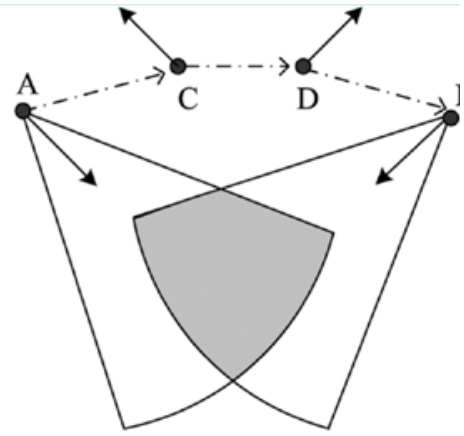

(b)
Figure 1. (a) FoV. (b) Overlapped FoV.

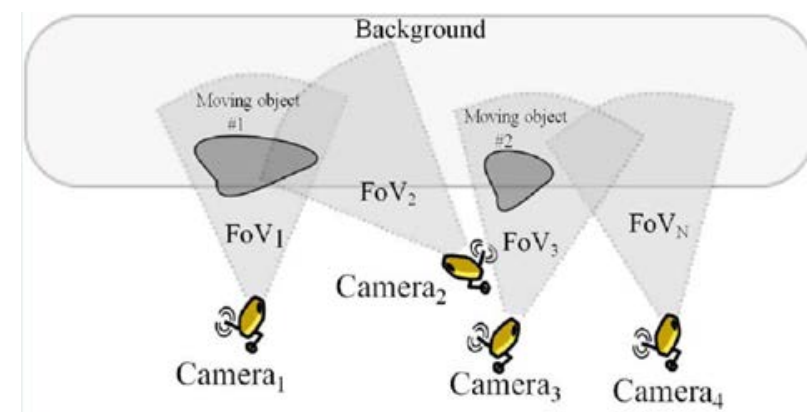

Figure 2. An example scenario of WMSN.

Each camera sensor node knows its own coordinates and orientation through an initial calibration phase. The next issue is to determine an optimum coverage of the sensing area. The sensors' coverage area depends on characteristics of the camera such as position, orientation, and angular view depth ${ }^{[9]}$. In WMSN, geographically neighbor nodes do not necessarily sense adjacent visual areas. On the other hand, two camera sensors can sense similar parts of the visual environment while their geographical distance could be greater than sensing radius. The same object may be seen from different points of view and at different depths by different cameras ${ }^{[10]}$.

Because the constrained energy of sensor nodes, the residual energy of each sensor varies with time. A sensor becomes dead while its battery runs out due to computation or communication. Live sensors are employed to monitor an area. Each sensor node is at a different coordinate with a different orientation. Hence, each subset of sensors has its own coverage area. Our main goal is to provide the maximum coverage of the target region with the minimum number of sensor nodes, while maximizing the network's lifetime.

\section{COVERAGE CONTROL ALGORITHM}

There are two major problems in multimedia sensor networks. One is that large amounts of data are redundant because of the overlapping FoV and the information in sensing blanks cannot be acquired. The other is that energy consumption is increased if redundant sensors keep in an active state ${ }^{[11]}$. In this section, the proposed solution of the sensor coverage and life time problem is described in details.

\section{A. Random Sensor Distribution and Camera Calibration}

At first, a certain number of camera sensor nodes are randomly distributed over the sensing area. Then, it is followed by camera calibration stage. Camera calibration is required in order to inform the base station of the geographical position and the angular orientation of each sensor. When a sensor transmits a captured image, the base station knows exactly which part of the area is photographed by that specific sensor. Therefore, it is possible for the base station to select a number of sensor nodes based on some criteria.

\section{B. Sensor Selection and Coverage Optimization}

Sensor selection will be performed based on sensor priority with the aim of coverage optimization. The first step is to check if network alive or not. If the coverage of live sensors is less than a predefined threshold then network is assumed to be useless.

In the second step, the priority of each sensor node is determined. This priority defined by function $\mathrm{f}$, which depends on different parameters. It is expected that the performance of the solution depends on the nature of the priority function. It is assumed that the priority function of the ith sensor depends on the remaining energy $\mathrm{E}_{\mathrm{i}}$, amount of overlap FoV (OV), and the coverage of the live sensors at $j$ th time step, $\mathrm{C}\left(\mathrm{L}_{\mathrm{j}}\right)$.

$$
\text { Priority }_{\mathrm{i}}=\mathrm{f}\left(\mathrm{E}_{\mathrm{i}}, \mathrm{OV}\left(\{\mathrm{i}\} \cup \mathrm{S}_{\mathrm{j}}\right), \mathrm{C}\left(\mathrm{L}_{\mathrm{j}}\right)\right)
$$

Suppose $E_{p}, E_{t}$ and $E_{r}$ denote the energy consumption of a camera node to process, transmit and receive a single image frame respectively, and $\mathrm{E}$ is the initial energy. Consider a scenario where each node within the WMSN processes, transmits and receives one image frame, the overall processing, transmission or receiving cost is given by,

$$
\mathrm{E}_{\mathrm{c}}=\mathrm{E}_{\mathrm{p}}+\mathrm{E}_{\mathrm{t}}+\mathrm{E}_{\mathrm{r}}
$$

Then, $E_{i}=E-E_{c}=E-\left(E_{p}+E_{t}+E_{r}\right)$

In this function, $\mathrm{OV}$ is a set of points in the target region composed of the intersection of at least two sensors' coverage sets. On the other hand if $\mathrm{X}$ is an arbitrary set of sensors, then we define $\mathrm{OV}(\mathrm{X})$ to be $\mathrm{OV}(\mathrm{X})=\{\mathrm{y} \mid \mathrm{y} \in \mathrm{C}(\mathrm{m}) \cap \mathrm{C}(\mathrm{n}), \mathrm{m}, \mathrm{n} \in \mathrm{X}\}$

After assigning a priority to each live sensor, the sensor with the highest priority among the unselected ones is selected and its index is stored in $\mathrm{I}_{\mathrm{k}}$.

$$
\mathrm{I}_{\mathrm{k}}=\underset{\mathrm{i} \in \mathrm{L}_{\mathrm{j}}-\mathrm{S}_{\mathrm{j}}}{\arg \max }\left\{\text { Priority }_{\mathrm{i}}\right\}
$$


Then, the sensor is added to the selected sensors list $S_{j}$. The set $S_{j}$ contains sensors which provide adequate coverage. $S_{j}=S_{j} \cup\left\{I_{k}\right\}$

At each iteration of this algorithm sensor is selected based on its priority and added to $\mathrm{S}_{\mathrm{j}}$. Parameter $\mathrm{k}$ is the iteration number and $\mathrm{I}_{\mathrm{k}}$ indicates the index of a sensor based on the employed priority function Pr iority ${ }_{i}$.

The third step is to determine the adequate level of coverage. It is checked if the maximum possible coverage can be achieved or not. If the current coverage is less than the whole coverage of live sensors right at the beginning of network's operation, then the task is degraded to achieving an acceptable level of coverage.

The adequate level of coverage, adaptively changes with the network conditions. A list of sensors is sorted in a descending order based on the designated priorities. Sensors with the greatest priority are selected until adequate coverage is reached. There might be sensors in the $S_{j}$ with the coverage area that is a subset of the coverage of other sensors. In such a case, it is necessary to deselect such sensors for energy saving. The process of discovering and deselecting sensors with redundant coverage is stated as following. In order to determine adequate coverage, we first initial the set $S_{j}$ to be empty.

For each sensor $\mathrm{S}$ in the $\mathrm{S}_{\mathrm{j}}$,

if

Coverage(Selected_List $)=$ Coverage $($ Selected_List $-\mathrm{S})$

then deselect the sensor $\mathrm{S}$ from the $\mathrm{S}_{\mathrm{j}}$.

Eventually, the algorithm forms the set $S_{j}$ of the selected sensors.

\section{Routing and Transmission}

When the adequate coverage is reached, the next work is to find appropriate routes between selected sensors and the base station. The selected sensor nodes transmit their data to the base station through the pre-constructed routes. In WMSN, sensing nodes should forward their information to a base station with the cooperation of other nodes. Hence, a set of source nodes provide data for a single destination. In other words, we have only one destination (base station) and a number of data providers (i.e. sensor nodes). This situation is similar to multicasting. While in multicasting, a single source provides data for a set of receivers. With this similarity, multicasting algorithm is adapted for routing strategy as follows.

- The base station broadcasts a route request packet which includes the list of the selected sensor nodes. Sensor nodes that receive the route request packet add their IDs to the packet and re-broadcast it.

- If a selected sensor node receives the broadcasted route request, it builds up a route reply packet and forwards it back to the base station. This route reply packet contains information about the transmission path.
- When the base station receives route reply packets from all the selected nodes, it determines the best route for each sensing node and sends a packet to inform the nodes about their transmission paths.

Wireless multimedia sensor networks generally operate based on limited energy which causes an active sensor to die after a while. Therefore, the base station should periodically initiate the routing strategy to bypass dead sensors and reestablish collapsed routes.

\section{SIMULATION RESULTS}

For the simulations conducted in this paper, 200 sensor nodes are randomly distributed over an $100 \times 100 \mathrm{~m}^{2}$ rectangular area. The offset angles of each camera are randomly distributed over the interval $[-(\pi / 4),+(\pi / 4)]$. The initial energy of each camera is randomly selected between $\mathrm{E}_{\min }$ and $\mathrm{E}_{\max }$.

Three different combinations of $\mathrm{Ei}, \mathrm{OV}$, and $\mathrm{C}\left(\mathrm{L}_{\mathrm{j}}\right)$ in priority function are presented in this paper. In the maximum coverage area (MCA) selection method, sensors are selected based on their coverage area. In this method, a list of sensors is sorted based on their coverage area. Live sensors with the greatest coverage areas are selected from top of the list. In the MCA selection method, the coverage area of each sensor is considered separately. However, selection of two sensors with large visual overlaps should be avoided. This problem is considered in the other two methods. In the minimum lifetime and overlap (MLO) method, we consider both the remaining power and overlap of sensors. In the symmetric difference (SD) method, the symmetric difference between the covered area and the coverage of each live sensor is calculated. This definition results in assigning higher priority to a sensor with larger coverage and lower overlap.

In order to evaluate the proposed algorithms four criteria are used. These criteria are the percentage of coverage, the number of live sensors, number of the selected sensors, and percentage of overlap. Since our goal is to maximize the network's lifetime, the algorithm that maximizes the timecoverage is preferable. Percentage of coverage during network's lifetime is shown in table 1. Among these algorithms, SD prolongs network's lifetime while it has the highest coverage too.

TABLE I. COVERAGE OF THE ALGOTITHMS

\begin{tabular}{|l|c|c|c|}
\hline \multirow{2}{*}{ Coverage(\%) } & \multicolumn{3}{|c|}{ Time step of the algorithms } \\
\cline { 2 - 4 } & MCA & MLO & SD \\
\hline 90 & 580 & 760 & 800 \\
\hline 80 & 655 & 786 & 845 \\
\hline 70 & 688 & 820 & 886 \\
\hline 60 & 735 & 855 & 915 \\
\hline 50 & 760 & 876 & 945 \\
\hline
\end{tabular}

Table 2 shows the number of live sensors for three algorithms. These algorithms operate with the same number of live sensors during a few initial steps of their lifetimes. It 
takes some time for a number of sensors to die. Thereafter the number of live sensors differs for each algorithm.

TABLE II. NUMBER OF LIVE SENSORS OF THE ALGOTITHMS

\begin{tabular}{|l|c|c|c|}
\hline \multirow{2}{*}{ Time step } & \multicolumn{4}{|c|}{ number of live sensors of the algorithms } \\
\cline { 2 - 4 } & $\boldsymbol{M C A}$ & $\boldsymbol{M L O}$ & $\boldsymbol{S D}$ \\
\hline 200 & 108 & 114 & 105 \\
\hline 400 & 91 & 107 & 83 \\
\hline 600 & 70 & 92 & 62 \\
\hline 800 & 30 & 51 & 40 \\
\hline 1000 & 5 & 10 & 15 \\
\hline
\end{tabular}

Number of the selected sensors of the network's operation is shown in table 3. At the initial time, number of selected sensors of the MCA algorithm is lower than the other two algorithms since there is overlap among sensors' coverage sets.

TABLE III.

NUMBER OF SELECTED SENSORS OF THE ALGOTITHMS

\begin{tabular}{|l|c|c|c|}
\hline \multirow{2}{*}{ Time step } & \multicolumn{3}{|c|}{ number of selected sensors of the algorithms } \\
\cline { 2 - 4 } & $\boldsymbol{M C A}$ & $\boldsymbol{M L O}$ & $\boldsymbol{S D}$ \\
\hline 200 & 4 & 5 & 6 \\
\hline 400 & 4.6 & 5.2 & 5.2 \\
\hline 600 & 7.3 & 5.4 & 4.8 \\
\hline 800 & 9.5 & 6.9 & 5.5 \\
\hline 1000 & 1.8 & 3.4 & 3.7 \\
\hline
\end{tabular}

Table 4 illustrates percentage of overlap of three algorithms. Since in the selection phase of SD and MLO the amount of overlap is considered, it is expected that these algorithms produce less overlap in comparison with MCA.

TABLE IV.

OVERLAP OF THE ALGOTITHMS

\begin{tabular}{|l|c|c|c|}
\hline \multirow{2}{*}{ Time step } & \multicolumn{3}{|c|}{ Overlap(\%) of the algorithms } \\
\cline { 2 - 4 } & MCA & MLO & SD \\
\hline 200 & 74 & 50 & 41 \\
\hline 400 & 68 & 51 & 49 \\
\hline 600 & 57 & 57 & 53 \\
\hline 800 & 23 & 43 & 48 \\
\hline 1000 & 1 & 6 & 12 \\
\hline
\end{tabular}

\section{CONCLUSION}

In this paper, a novel coverage-enhancing algorithm based on sensors' priority is proposed in WMSN. The goal of the study is to maintain complete visual coverage of the area for the longest period of time. To form a complete coverage of the target plane, a group of sensors must be selected from an initially large number of randomly distributed sensors. Each sensor is assigned a priority based on its remaining energy, its coverage, and the overlap area. Among different probable combinations of these parameters that can produce different priority functions, we used three of them and through simulations we showed that these parameters have important roles in network lifetime. These priorities were coverage; overlap and remaining energy; and symmetric difference between each sensor coverage and previously covered area. We showed that the symmetric difference priority has superior performance compared to the other priorities.

\section{ACKNOWLEDGMENT}

This work was supported by Key Research and Development Program of Jiangxi Province (20161BBG70040), Science and Technology Project of Jiangxi Department of Education (GJJ151188, GJJ151199), Science and Technology Landing Project of Jiangxi University (KJLD13098).

\section{REFERENCES}

[1] I. F. Akyildiz, T.Melodia, and K.R. Chowdhury, "A survey on wireless multimedia sensor networks," Computer Networks, 2007, vol.51, no.4, pp.921-960.

[2] B. Wang, "Coverage problems in sensor networks: A survey," ACM Comput. Surv., vol. 43, no. 4, pp. 1-53, Oct. 2011.

[3] E. Yildiz, K. Akkaya, E. Sisikoglu, M. Sir, and I. Guneydas, "Camera deployment for video panorama generation in wireless visual sensor networks,” in Proc. Int. Symp. Multimedia, Dec. 2011, pp. 595-600.

[4] H. Li, V. Pandit, and D. P. Agrawal, "Gaussian distributed deployment of relay nodes for wireless visual sensor networks," in Proc. IEEE Global Commun. Conf., Dec. 2012, pp. 5374-5379.

[5] D. Tao, H. D. Ma, and L. Liu, "A virtual potential field based coverageenhancing algorithm for directional sensor networks," J. Softw., vol. 18, no. 5, pp. 1152-1163, May 2007.

[6] H. Huang, L. Sun, R. Wang, and J. Li, "A novel coverage enhancement algorithm for image sensor networks," Int. J. Distrib. Sens. Netw., vol. 2012, no. 370935, pp. 1-11, Mar. 2012.

[7] R. Dai and I. F. Akyildiz, "A spatial correlation model for visual information in wireless multimedia sensor networks," IEEE Trans. Multimedia, 2009, vol.11, no.6, pp.1148-1159.

[8] Jian Chen, Lu Zhang, and Yonghong Kuo, "Coverage-Enhancing Algorithm Based on Overlap-Sense Ratio in Wireless Multimedia Sensor Networks," IEEE Sensors Journal, vol. 13, no. 6, June 2013, pp.2077-2083.

[9] Subir Halder and Amrita Ghosal. "A Location-Wise Predetermined Deployment for Optimizing Lifetime in Visual Sensor Networks," IEEE Trans. Circuits Syst. Video Technol., vol. 26, no. 6, June 2016, pp. 1131-1145.

[10] Z. Sheng, J. Fan, C. H. Liu, V. C. M. Leung, X. Liu, and K. K. Leung, Energy-efficient relay selection for cooperative relaying in wireless multimedia networks,” IEEE Trans. Veh. Technol., vol. 64, no. 3, pp. 1156-1170, Mar. 2015.

[11] Stefania Colonnese,Francesca Cuomo,and Tommaso Melodia,"An Empirical Model of Multiview Video Coding Efficiency for Wireless Multimedia Sensor Networks,"IEEE Trans. Multimedia, vol. 15, no. 8, December 2013, pp.1800-1814. 